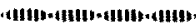

Note

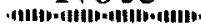

(Received 20 July, 1997 ; Accepted 6 October, 1997)

\section{Observation on Skin-Core, Microfibril and Pleated Band Morphology in Poly (p-phenylene Terephthalamide) Fibers Using Transmission and Scanning Electron Microscopes}

\author{
Takashi Itoh and Masato Hashimoto
}

Department of Polymer Science and Engineering, Faculty of Textile Science, Kyoto Institute of Technology, Kyoto, 606 Japan

\begin{abstract}
Poly (p-phenylene terephthalamide) fibers (Kevlar 29 and 49) were thin-sectioned or treated with a mixed solvent of sulfuric-acid/water (SAW) to be observed in transmission and/or scanning electron microscopes. Microfibrillar and pleated-band morphology existed in Kevlar 29 and 49 fibers, while the skin-core structure was confirmed only in Kevlar 29 fiber. A microfibril ca. $100 \mathrm{~nm}$ thick is composed of several tens of needle-shaped microcrystals $10 \sim 20 \mathrm{~nm}$ thick, being pleated with a period of $500 \sim 600 \mathrm{~nm}$ along the fiber axis. Hydrogen-bonding or crystal structure in the core region was considered to be more ordered than that in the skin region in the case of Kevlar 29 fiber. Orientation of microcrystais in each microfibril was incoherent although $b$-axis orientation mainly distributed around the radial direction. Such tendency is more prominent for Kevlar 29 fiber which was finally suggested to assume the hierarchical morphology of "pleated-microcrystal band".
\end{abstract}

\section{Introduction}

Several basic studies have been reported for crystal and morphological structures of poly (p-phenylene terephthalamide) (PPTA) fibers[1-12]. The epochmaking works have been performed by Yabuki et al. $[2,4]$ and Dobb et al. [5] for the morphological features of the PPTA fiber. The former workers suggested that the PPTA fiber, which was prepared in their laboratory following the method to make Kevlar fiber (a trademark of E. I. du Pont de Nemours \& Co.), comprised pillar-shaped microfibrils and the orientation of the b-axes of crystals is radial in the sheath (skin) and random in the core, while the latter indicated on the basis of the electron diffraction and dark-field image from Kevlar 49 fiber that the supermolecular architecture of the fiber consisted of a system of sheets regularly pleated along the long axis and arranged radially. Dobb et al., however, did not succeed in observing the pleated sheet morphology directly in the bright-field electron microscopic image nevertheless they tried to find it in the fiber fragments produced by ultrasonic dispersion, and did not refer to the microfibril and skin-core structures so explicitly. Such variation in the experimental results for different PPTA fibers seems to suggest that the morphological structure in PPTA fiber may depend on the fabrication process. We, however, have not found the study which explicitly examines and discusses the morphological difference between different PPTA fibers.

In this study, Kevlar 29 and 49 fibers were treated with sulfuric-acid/water (SAW) mixed solvent at elevated temperatures to be dispersed into fragments, and then observed in transmission and scanning electron microscopes. The morphological aspects of the different PPTA fibers were examined on the basis of the results for the SAW-treated specimens, being compared with the electron diffractions and dark-field images taken from thin sections of the fibers. 


\section{Experimental}

Kevlar 29 and 49 were used as the original PPTA fibers. Kevlar 29 fiber was annealed at 573 and $673 \mathrm{~K}$ for $30 \mathrm{~min}$ in vacuum. These fibers (original Kevlar 49 and annealed Kevlar 29) were subsequently kept in sealed glass tubes at $463 \mathrm{~K}$ for $15 \mathrm{~min}$, which contained mixed solvent of sulfuric-acid/water (SAW) $\left(\mathrm{H}_{2} \mathrm{SO}_{4}\right.$ : $\mathrm{H}_{2} \mathrm{O}=7: 3$ in volume). The original (not annealed) Kevlar 29 fiber was also SAW- treated at $458 \mathrm{~K}$ for 10 min and subsequently dispersed by ultrasonic radiation for $30 \mathrm{~s}$. These SAW-treated specimens were observed in a scanning electron microscope (SEM: Hitachi S-410) after Au coating as well as in a transmission electron microscope (TEM: JEOL, JEM-100C).

Thin longitudinal sections ca. $100 \mathrm{~nm}$ thick were cut in a direction (moving direction of the knife) perpendicular to the fiber axis using an ultramicrotome (Sorvall, MT-1) with a sapphire knife after embedment in epoxy resin and then picked up on copper grids to be examined in the TEM. The electron diffraction patterns were recorded from specimen (section) areas of diameter ca. $2 \mu \mathrm{m}$ by means of a selected area aperture.

\section{Results and Discussion}

A SEM photograph of Kevlar 29 fiber is shown in Fig. 1(a), which was annealed at $673 \mathrm{~K}$ for $30 \mathrm{~min}$ in vacuum and then SAW-treated, while the SAW-treated Kevlar 49 original (not annealed before SAW. treatment) fiber is shown in Fig. 1 (b). A cylindrical rod (C) appears to be laid on the broken sheath or layer (S) surrounding the rod halfway in the case of Kevlar 29 fiber (Fig. 1(a)). Similar structure was also observed in other SAW-treated Kevlar 29 fibers (original and annealed at $573 \mathrm{~K}$ before SAW-treatment). It should be noted that diameter of the original Kevlar 29 fiber is $13.1 \mu \mathrm{m}[8]$, while the rod diameter observed for the SAW-treated Kevlar 29 is $8 \sim 9 \mu \mathrm{m}$, which suggests that Kevlar 29 fiber consists of skin (ca. $2 \sim 2.5 \mu \mathrm{m}$ thick) and core regions, qualitatively supporting the morphological model proposed by Yabuki et al. (4) for PPTA fiber prepared for themselves. The figure also suggests that the explicit boundary does exist between skin and core regions which seem to have different
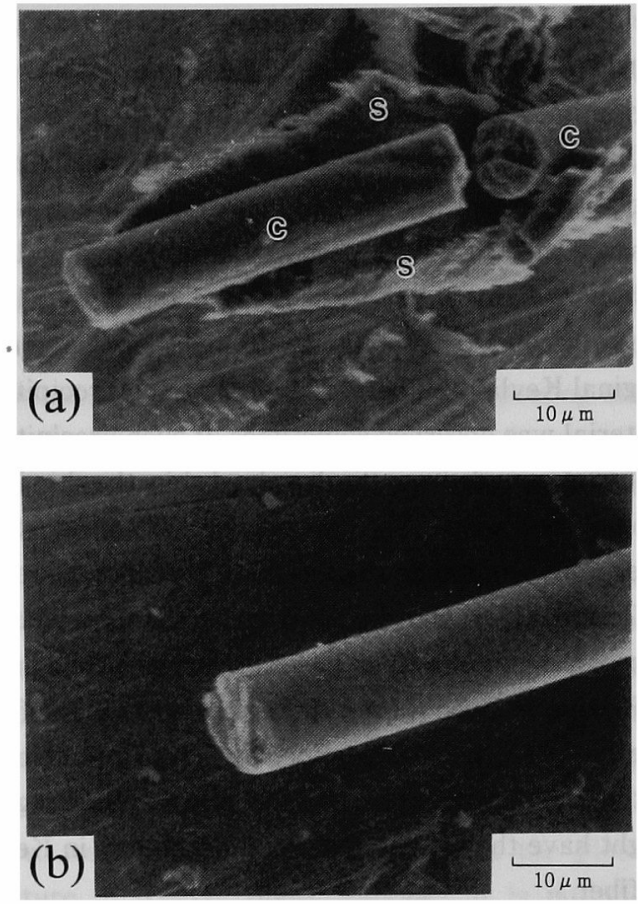

Fig. 1 SEM photographs of (a) Kevlar 29 fiber annealed at $673 \mathrm{~K}$ for $30 \mathrm{~min}$ in vacuum and (b) original Kevlar 49 fiber. Both fibers were treated with SAW solvent $\left(\mathrm{H}_{2} \mathrm{SO}_{4}: \mathrm{H}_{2} \mathrm{O}=7: 3\right.$ in volume) at $463 \mathrm{~K}$ for $15 \mathrm{~min}$ before observation. Skin-core structure is observed explicitly in Kevlar 29 fiber. C: core, S: skin.

resistance to the SAW solvent although Yabuki et al. (4) assumed the continuous change in the b-axis orientation of the crystallites along the radial axis of the fiber through the birefringence calculation. Structural difference between skin and core regions of PPT A fiber has been proposed by Morgan et al. [9]; the skin layer exists in PPTA fiber, where the chain ends are arranged or staggered randomly relative to one another along the fiber axis, while those become more ordered in the core region, resulting in periodic transverse weak planes every ca. $200 \mathrm{~nm}$ or average PPTA molecular length. They have suggested that the skin region is less crystalline than the core region because the exterior of the PPTA- $\mathrm{H}_{2} \mathrm{SO}_{4}$ dope is cooled at a significantly faster rate than the interior upon solidification of the dope in the coagulation bath. They have claimed that such suggestion is supported by diffuse electron diffraction pattern from the exterior of 
the fiber relative to the fiber core. Low crystallinity is considered to reduce the acid-resistance of the skin region in the case of Kevlar 29 fiber.

On the other hand, such characteristic morphology explicitly indicating the skin-core structure was not observed in the SAW-treated Kevlar 49 fiber (Fig. 1(b)), although diameter of the SAW-treated fiber (10 $12 \mu \mathrm{m})$ seems to be a little smaller than that of the original Kevlar 49 fiber $(12.6 \mu \mathrm{m}[8])$ and precipitated material was observed sometimes. If such precipitated material is made from the dissolved skin, the skin layer should be very thin in Kevlar 49 fiber. Such interpretation is not considered to contradict the report of Dobb et al. [5] who did not mention the skin structure except the oxidized fiber, based on the dark-field image of Kevlar 49. Even if the skin region exists in the fiber, it might be too thin to be defined by the dark-field image of the thin section, or both of the skin and core regions might have the similar pleated morphology in Kevlar 49 fiber.

The morphological difference between the SAW treated Kevlar 29 and 49 fibers suggests that the further drawing process of ca. $0.5 \%$ at $823 \mathrm{~K}$ for $1-6 \mathrm{~s}$ during fabrication of Kevlar 49 causes some changes in structure and physical properties of Kevlar 49 fiber in comparison with those of Kevlar 29 fiber. Morgan et al. (9) have reported that Kevlar 49 fiber is not completely dissolved in $100 \%$ sulfuric acid at $333 \mathrm{~K}$ even at low PPTA concentrations $<5 \mathrm{wt} \%$, while Kevlar 29 completely dissolves in the same condition.

The initial modulus of the original Kevlar 29 fiber was estimated from the stress-strain curve to be $0.5 \times 10^{11} \mathrm{~Pa}$ and that of the original Kevlar 49 fiber was $1 \times 10^{11} \mathrm{~Pa}$ for Kevlar 49. It should be noted that the initial moduli of Kevlar 29 fibers annealed at $573 \mathrm{~K}$ and 673 $\mathrm{K}$ without constraint are almost the same as that of the original Kevlar 29 fiber. On the basis of such aspects for structure and physical property of the original and annealed Kevlar 29 fibers, annealing without tension does not seem to cause so serious change in PPTA fiber. In other words, drawing process at an extremely high temperature during fabrication of Kevlar 49 may increase the structural order and/or the degree of the molecular orientation along the fiber axis in comparison with Kevlar 29 fiber, which seems to reflect on such high modulus of Kevlar 49 fiber.
Diffraction patterns and dark-field TEM images of Kevlar 29 fiber annealed at $673 \mathrm{~K}$ for $30 \mathrm{~min}$ in vacuum and the original Kevlar 49 fiber are shown in Fig. 2. The dark-field image was formed selecting one of the off-meridional reflections on the sixth layer which was apparently indexed as 106 by Dobb et al. (5) Judging from width of these sections $(5 \sim 7 \mu \mathrm{m})$, the sections were cut from the surface regions of the fibers ca. $13 \mu \mathrm{m}$ in diameter.

The 200 diffraction is generally stronger than 110 one suggesting the radial orientation of b-axis in the crystal because the electron beam is parallel to the radial direction of the fiber in the surface section. Intensity of 200 reflection is sometimes comparable with 110 one for Kevlar 29 (e.g. Fig. 2(a)), which suggests broader distribution of b-axis orientation in Kevlar 29 fiber than in Kevlar 49 fiber.

The off-meridional reflections on the sixth layer
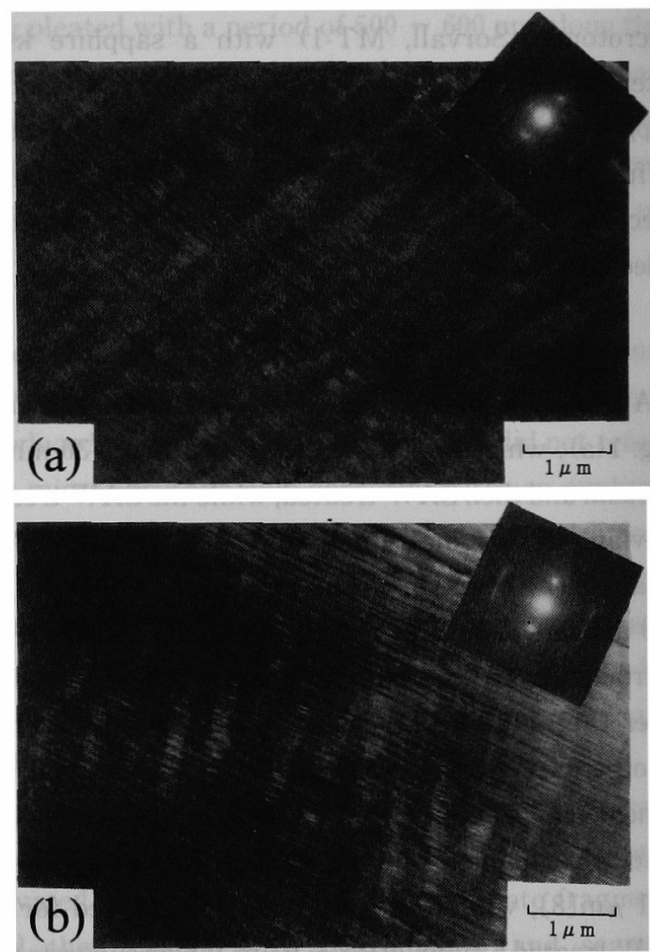

Fig. 2 Diffraction patterns and dark-field TEM images from sections of (a) Kevlar 29 fiber annealed at $673 \mathrm{~K}$ for $30 \mathrm{~min}$ in vacuum and (b) original Kevlar 49 fiber. Dark-field images were formed selecting one of the offmeridional reflections on the sixth layer line. 
explicitly split in Kevlar 49 while such splitting is not so clear generally in the case of Kevlar 29 fiber. Although Dobb et al. [5] indexed such off-meridional reflections as apparent 106 , the splitting angle is $20-25^{\circ}$, which seems to contradict the crystal structure of PPTA fiber reported by Northolt [1] where the splitting angle between 106 reflections is to be $30-31^{\circ}$. In addition, intensity of 006 reflection is to be stronger than that of 106 reflection according to the structure analysis of Northolt $[1]$. The orientational distribution of the $\mathrm{c}$-axis pleated around b-axis is judged from the azimuthal width of 200 reflection, the half-height width of which is estimated to be $10-20^{\circ}$, corresponding to the equatorial width or splitting angle of the reflection on the second layer which are regarded as 002 reflection because intensity of the 102 reflection is negligible[1]. Considering these facts on the basis of the crystal structure of PPTA fiber[1], a possible explanation is that the off-meridional reflections on the sixth layer may be superposition of the splitted 006 and 106 reflections. If it is the case, the splitting-angle variation depending on the observed position is explained by the difference in the orientational distribution of the c-axis, and the unclear splitting of the reflection from Kevlar 29 fiber may be due to the broad distribution of the $c$-axis orientation or chain pleating in the fiber, although such interpretation is not conclusive at present. More precise and quantitative analysis is needed for the diffraction profiles of Kevlar fibers.

The obtained dark-field image of Kevlar 49 (Fig. 2 (b)) is intrinsically the same as that reported for the nondiametric section of Kevlar 49 by Dobb et al. [5], where broad bands of spacing, approximately $500 \mathrm{~nm}$, are observed. Similar band structure also appears in Kevlar 29 as shown in Fig. 2(a) although the band period (ca. $600 \mathrm{~nm}$ ) is a little larger than that of Kevlar 49. The characteristic feature in the dark-field image is microfibrillar morphology, thickness of which is $100 \mathrm{~nm}$ order, in which needle-shaped bright domains $10 \sim 20$ $\mathrm{nm}$ thick and $200 \sim 300 \mathrm{~nm}$ long are observed, reflecting the coherent crystalline regions. Concept of microfibril has been suggested to exist by Yabuki et al[2], based on the small-angle $\mathrm{X}$-ray scattering, which seems to correspond to the microfibrillar structure ca. $100 \mathrm{~nm}$ thick observed in the dark-field images, while Dobb et al. have not referred to the microfibril structure in
Kevlar 49[5].

The microfibril is definitely observed in the bright-field images of Kevlar 29 fibers as shown in Fig. 3, which becomes more explicit when the annealing temperature increases (compare Fig. 3 (a) with Fig. 3 (b); annealing temperatures are 573 and $673 \mathrm{~K}$ respectively). Such development of the microfibrillar texture, however, does not seem to be advantageous for mechanical property of the fiber because peeling off of the surface layer is always observed in the annealed Kevlar fiber, which also suggests weak interaction between the microfibrils.

A bright-field TEM image of fragments of the SAW-treated (at $458 \mathrm{~K}$ for $10 \mathrm{~min}$ ) and subsequently dispersed (by ultrasonic radiation) Kevlar 29 (original or not annealed) fiber is shown in Fig. 4, where the pleated structure is explicitly observed. Since the period of pleating is similar to that in the dark-field image, we consider it one evidence of the pleated structure in Kevlar fibers although it is not clear whether this fragment comes from skin or core region. It is also noted that the microfibrillar texture is ambiguous and observed partly in the fragments. It is possible that the microfibrils collapsed during the

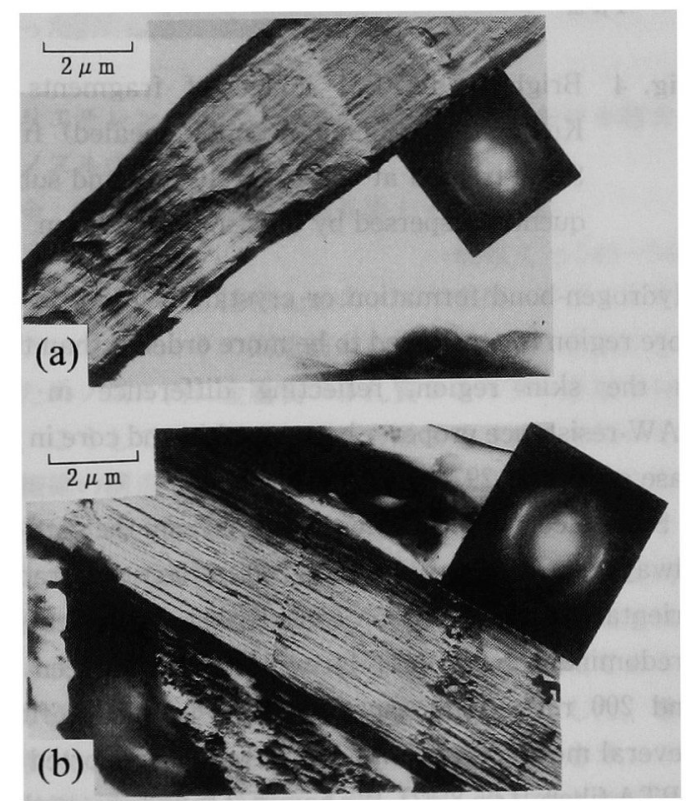

Fig. 3 Bright-field TEM images and diffraction patterns taken from sections of Kevlar 29 fibers annealed at (a) $573 \mathrm{~K}$ and (b) $673 \mathrm{~K}$. 
SAW-treatment and/or ultrasonic dispersion procedures.

Summarizing the results, microfibrillar and pleated morphology was confirmed to exist in both Kevlar 29 and 49 fibers; the former had been suggested by Yabuki et al[2] in the self-prepared PPTA fiber and the latter had been proposed by Dobb et al. [5] in Kevlar 49 fiber. A microfibril ca. $100 \mathrm{~nm}$ thick is composed of several tens of needle-shaped microcrystals $10 \sim 20 \mathrm{~nm}$ thick which are pleated with a period of $500 \sim 600 \mathrm{~nm}$ along the fiber axis. On the other hand, the skin-core structure with the definite boundary was confirmed only in Kevlar 29 fiber, which suggests that some morphological difference may exists in the skin-core morphology between Kevlar 29 and 49 fibers; the skin layer in Kevlar 49 fiber might be too thin to be observed.

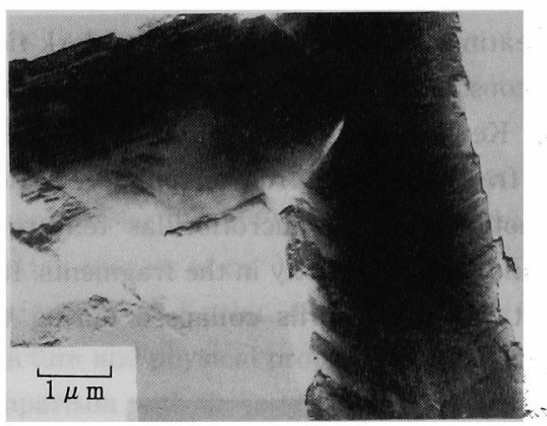

Fig. 4 Bright-field TEM image of fragments of Kevlar 29 (original or not annealed) fiber SAW-treated at $458 \mathrm{~K}$ for $10 \mathrm{~min}$ and subsequently dispersed by ultrasonic radiation.

Hydrogen-bond formation or crystal structure in the core region is considered to be more ordered than that in the skin region, reflecting difference in the SAW-resistance property between skin and core in the case of Kevlar 29 fiber.

Since electron diffraction patterns from the sections always show both 110 and 200 reflections, radial orientation of $\mathrm{b}$-axis is not considered to be perfect but predominant, based on relative intensity between 110 and 200 reflections, especially for Kevlar 29 fiber. Several morphological models have been reported for PPT A fibers. $[4,5,8-12]$. We have not found any explicit morphological evidence to show the skin-core morphology of Kevlar 49 fiber in the previous studies, while the experimental results suggesting the skin-core structure have been presented for Kevlar 29[10] or self-prepared PPTA $[2,4]$ fiber where the skin is a few $\mu \mathrm{m}$ thick. Such situation seems to be consistent with our result.

It has been also reported(12) that the longitudinal cleavage planes with cylindrical surface around fiber axis exist in the core region of Kevlar 29 fiber. Such a fact and broad distribution of the radial $b$-axis orientation even in the skin region of Kevlar 29 fiber seem to be contrary to the conception of the radially arranged "pleated sheet" proposed by Dobb et al. [5] $\mathrm{We}$, therefore, are afraid that naming of the "pleated sheet" might not reflect the morphology in Kevlar 29 fiber properly, where crystalline orientation of the needle-shaped microcrystals is independent or incoherent although $\mathrm{b}$-axis orientation mainly distributes around the radial direction. The "pleated-microcrystal band" might be more appropriate expression on the morphology of PPTA fiber, especially for Kevlar 29 fiber.

\section{References}

1. M. G. Northolt, European Polymer J., 10, 799 (1974).

2. K. Yabuki, H. Ito, and T. Ota, Sen'i Gakkaishi, 31, T524 (1975)

3. M. G. Dobb, A. M. Hindeleh, D. J. Johnson, and B. P. Saville, Nature, 253, 189 (1975).

4. K. Yabuki, H. Ito, and T. Ota, Sen'i Gakkaishi, 32, T55 (1976).

5. M. G. Dobb, D. J. Johnson, and B. P. Saville, J. Polym. Sci. Polym. Phys., 15, 2201 (1977).

6. M. G. Dobb, D. J. Johnson, A. Majeed, and B. P. Saville, Polymer, 20, 1284 (1979).

7. L. L. Chapoy, D. Spasenka, and K. Rasmussen, Macromolecules, 12, 680 (1979).

8. S. Manabe, S. Kajita, and K. Kamide, Sen'i Kikai Gakkaishi, 33, T93(1980).

9. R. J. Morgan, C. O. Pruneda, and W. J. Steele, J. Polym. Sci. Polym. Phys., 21, 1757 (1983).

10. L.-S. Li, L. F. Allard, and W. C. Bigelow, J. Macromol. Sci. Phys., B22, 269(1983).

11. M. Panar, P. Avankin, R. C. Blume, K. H. Gardner, T. D. Gierke, and H. H. Yang, J. Polym. Sci. Polym. Phys., 21, 1955(1983).

12. M. Horio, T. Kaneda, S. Ishikawa, and K. Shimamura, Sen'i Gakkaishi, 40, T285(1984). 